\title{
NGTS Observations of Enhanced Nanoflare Activity in Fully Convective Stars Linked to Plasma Resistivity
}

Christopher Dillon ( $\nabla$ cdillon06@qub.ac.uk)

Queen's University Belfast https://orcid.org/0000-0003-2709-7693

David Jess

Queen's University Belfast https://orcid.org/0000-0002-9155-8039

Mihalis Mathioudakis

Queen's University Belfast https://orcid.org/0000-0002-7725-6296

Chris Watson

Queen's University Belfast

James Jackman

Arizona State University

Peter Wheatley

University of Warwick

Michael Goad

University of Leicester

Sarah Casewell

University of Leicester

David Anderson

University of Warwick

Matthew Burleigh

University of Leicester

Samuel Grant

Queen's University Belfast https://orcid.org/0000-0001-5170-9747

Richard West

University of Warwick

José Vinés

Universidad de Chile

\section{Article}

Keywords: nanoflare activity, M-dwarf stars, MV stars 
Posted Date: September 24th, 2021

DOl: https://doi.org/10.21203/rs.3.rs-882520/v1

License: (c) (i) This work is licensed under a Creative Commons Attribution 4.0 International License. Read Full License 


\title{
NGTS Observations of Enhanced Nanoflare Activity in Fully Convective Stars Linked to Plasma Resistivity
}

\author{
C. J. Dillon ${ }^{1, *}$, D. B. Jess ${ }^{1,2}$, M. Mathioudakis ${ }^{1}$, C. A. Watson ${ }^{1}$, J. A. G. Jackman ${ }^{3}$, \\ P. J. Wheatley ${ }^{4}$, M. R. Goad ${ }^{5}$, S. L. Casewell ${ }^{5}$, D. R. Anderson ${ }^{4}$, M. R. Burleigh ${ }^{5}$, \\ S. D. T. Grant ${ }^{1}$, R. G. West ${ }^{4}$, and J. I. Vines ${ }^{6}$ \\ ${ }^{1}$ Astrophysics Research Centre, School of Mathematics and Physics, Queen's University Belfast, Belfast, BT7 1NN,
UK
${ }^{2}$ Department of Physics and Astronomy, California State University Northridge, Northridge, CA 91330, USA
${ }^{3}$ School of Earth and Space Exploration, Arizona State University, Tempe, AZ, 85287, USA
${ }^{4}$ Department of Physics, University of Warwick, Gibbet Hill Road, Coventry CV4 7AL, UK
${ }^{5}$ School of Physics and Astronomy, University of Leicester, University Road, Leicester, LE1 7RH, UK
${ }^{6}$ Departamento de Astronomia, Universidad de Chile, Casilla 36-D, Santiago, Chile
'cdillon06@qub.ac.uk
}

15 ABSTRACT

Previous examination of fully-convective M-dwarf stars highlighted unexplained enhanced rates of nanoflare activity. A potential explanation was linked to the helical turbulence dynamo which operates in fully convective stars. However, recent studies have found this helical dynamo does not appear significantly different to the Solar dynamo. The specific role the convective boundary plays on observed nanoflare rates, until now, was not known. Here we find evidence that fully convective M2.5V (and later) 6 stars display greatly enhanced nanoflare rates compared with their pre-convective boundary counterparts. Importantly, the rate of nanoflare activity increases with increasing spectral sub-type, with nanoflares exhibiting greatly enhanced flaring rates via Sweet-Parker reconnection. This occurs more favourably at increased plasma resistivities experienced in these later MV stars, suggesting a direct interplay between the rate of nanoflare occurrence and the intrinsic plasma parameters. As such, nanoflare behaviour is likely to be unrelated to the behaviour of the local dynamo.

\section{Introduction}

Nanoflares are small-scale magnetic reconnection events, with individual energies around $10^{9}$ times less than their large-scale counterparts, spanning between $10^{22}$ to $10^{25}$ ergs. Following magnetic reconnection, impulsive energy deposition heats the local plasma ${ }^{1-3}$. This leads to the impulsive brightening of the plasma ${ }^{4}$, which is followed by a decrease in the emitted radiation due to subsequent plasma cooling. The $e$-folding time, $\tau$, is the time taken for the flare luminosity to decrease by a factor $1 / e$, with its magnitude governed by the underlying local plasma conditions, such as the efficiencies of evaporative, non-evaporative, conductive, and radiative cooling processes ${ }^{5}$. Solar and stellar flare energies are governed by a power-law relationship ${ }^{6}$. Here, the power-law exponent governs the frequency, $d N / d E$, of flaring events with an associated energy, $E$, through the relationship,

$$
\frac{d N}{d E} \propto E^{-\alpha}
$$

where $\alpha$ represents the power-law index. The expected nature of the power-law relation for flares implies that low-energy flares should be many times more frequent than larger events, and that small-scale events will become more energetically significant as the power-law index, $\alpha$, increases. As a result, the occurrence rates of nanoflares are expected to be orders of magnitude more frequent than macroscopic flares. Indeed, nanoflare rates could be as high as several thousand per second over an entire stellar surface 7,8 , which would make them a considerable component of the stellar energy budget. As a result, nanoflares have been theorised as a solution to a long standing energy problem in astrophysics; the coronal heating paradox.

This paradox is that the outermost layer of the solar atmosphere is millions of degrees hotter than preceding layers, with no conclusive mechanism found to explain this heating ${ }^{9}$. Flares occur primarily in the corona ${ }^{10}$, so would be able to drive local heating via in-situ magnetic reconnection. However, the occurrence of large-scale flaring events are too infrequent to supply 
sufficient energy to the corona. Nanoflares, on the other hand, have been proposed as a series of small-scale impulsive events driving coronal heating that, as a consequence of their low individual energies, had been hither-to undiscovered - explaining why the source of coronal heating may have remained unobserved ${ }^{7,11-13}$.

This potential holds for stellar atmospheres as well as for the Sun. While solar nanoflares have been a considerable topic of interest over the last 20 years, stellar nanoflares have been almost entirely unexplored - remaining stubbornly below the noise floor of observations, with their low energies precluding the identification of individual stellar nanoflare events. However, nanoflares exhibit statistical signatures that distinguish them from the quiescent background of a star, alongside characteristic quasi-periodic signatures ${ }^{8,14}$. The statistical signatures manifest themselves as distinct offsets from the normal distribution expected of shot noise patterns, which can be benchmarked by studying successive intensity fluctuations of the stellar time series. The periodic signatures manifest as features present in Fourier spectral energy densities, namely spectral slopes, an associated 'turning-point' where the spectral slope begins, and clear peaks in the range $1-10 \mathrm{mHz}$. These techniques do not rely on the individual identification of nanoflare events, so can identify nanoflare activity in seemingly quiescent time series ${ }^{8}$.

Such techniques have been applied to time series of seemingly quiescent, fully convective M-dwarf stars, uncovering novel evidence of stellar nanoflare activity ${ }^{8}$. A combination of statistical and Fourier analyses were used to examine both observational lightcurves and synthetic time series with simulated nanoflare signals. This allowed for the diagnosis of underlying nanoflare activity rates in these stars, finding stellar nanoflare activity in an M3V and two M2.5V stars. Part of this evidence was heightened (with respect to previous solar and stellar investigations) power-law slopes when the nanoflare occurrence rates were plotted as a function of their underlying energy. These power-law slopes were in excess of $\alpha=3$, meaning that nanoflare activity forms a significant component of the stars overall energy budget. Here, we develop and apply these proven statistical nanoflare analysis techniques to a wide range of M-type stars. We examine 5 stars for each spectral sub-type, consisting of M0V, M1V, M2V, M2.5V, M3V, and M4V, spanning either side of the predicted convective boundary, which recent studies have placed at approximately $\mathrm{M} 2.1-2.3 \mathrm{~V}^{15}$. We compare their statistical and Fourier properties to previously generated nanoflare simulations ${ }^{8}$ in order to determine the underlying nanoflare conditions and the effect of the convective boundary on the uncovered nanoflare properties.

\section{Results}

\section{Statistical Analysis}

We present two example histograms of statistical intensity fluctuations in Figure 1 for stars observed with the Next Generation Transit Survey (NGTS - see Methodology for a description of the observations). These are NGTS J052346.3-361114 (M0V spectral type; top panel) and NGTS J050423.8-373021 (M4V spectral type; lower panel), where each time series has been detrended, with resulting intensity fluctuations normalised by the associated standard deviation, $\sigma_{N}$. From Figure 1 it is clear that opposite ends of the included spectral types, which lie on either side of the predicted convective boundary, demonstrate distinctly different statistical signatures. The M0V star exhibits very weak and inconclusive nanoflare signatures, with minimal negative median offset of the histogram and no elevated intensity fluctuations at $\sim 2 \sigma_{N}$. On the contrary, the M4V star has a clear excess of $\sim 2 \sigma_{N}$ intensity fluctuations, in addition to a prominent negative offset of the median histogram occurrence. The signatures of the M4V star shown in Figure 1 are consistent with previous positive stellar nanoflare identifications ${ }^{8}$. The distinct increase of visible nanoflare signatures within the expected regime of full convection is a promising sign that the enhanced nanoflare rates are related to the underlying convective nature of the star.

These examples illustrated in Figure 1 clearly identify the vastly different nanoflare signatures present at either end of the investigated range of spectral sub-types. To better examine the change in nanoflare activity across the given spectral range (M0V $-\mathrm{M} 4 \mathrm{~V})$, the derived properties were averaged according to their specific spectral type following bootstrap methods ${ }^{16}$. Traditional averaging of features that are dependent on the underlying stellar plasma conditions from multiple stars is challenging due to the unpredictability of the standard errors of the given parameters. Hence, bootstrapping techniques are used extensively throughout the physical sciences to better calculate confidence intervals for data following non-standard or unknown distributions ${ }^{17-19}$.

\begin{tabular}{|l|l|l|l|l|}
\hline Spectral Type & Median offset $\left(\sigma_{N}\right)$ & Fisher Skewness & $\zeta$ ratio & Kurtosis \\
\hline M0V & $-0.040 \pm 0.001$ & $0.004 \pm 0.002$ & $1.740 \pm 0.005$ & $0.128 \pm 0.010$ \\
\hline M1V & $-0.040 \pm 0.001$ & $0.008 \pm 0.004$ & $1.746 \pm 0.008$ & $0.180 \pm 0.013$ \\
\hline M2V & $-0.030 \pm 0.001$ & $0.003 \pm 0.011$ & $1.766 \pm 0.010$ & $0.196 \pm 0.037$ \\
\hline M2.5V & $-0.050 \pm 0.000$ & $0.019 \pm 0.003$ & $1.739 \pm 0.004$ & $0.227 \pm 0.072$ \\
\hline M3V & $-0.050 \pm 0.000$ & $0.025 \pm 0.004$ & $1.750 \pm 0.006$ & $0.180 \pm 0.017$ \\
\hline M4V & $-0.050 \pm 0.000$ & $0.051 \pm 0.014$ & $1.754 \pm 0.010$ & $0.267 \pm 0.029$ \\
\hline
\end{tabular}

Table 1. Averaged characteristics of the statistical properties by each spectral type. 


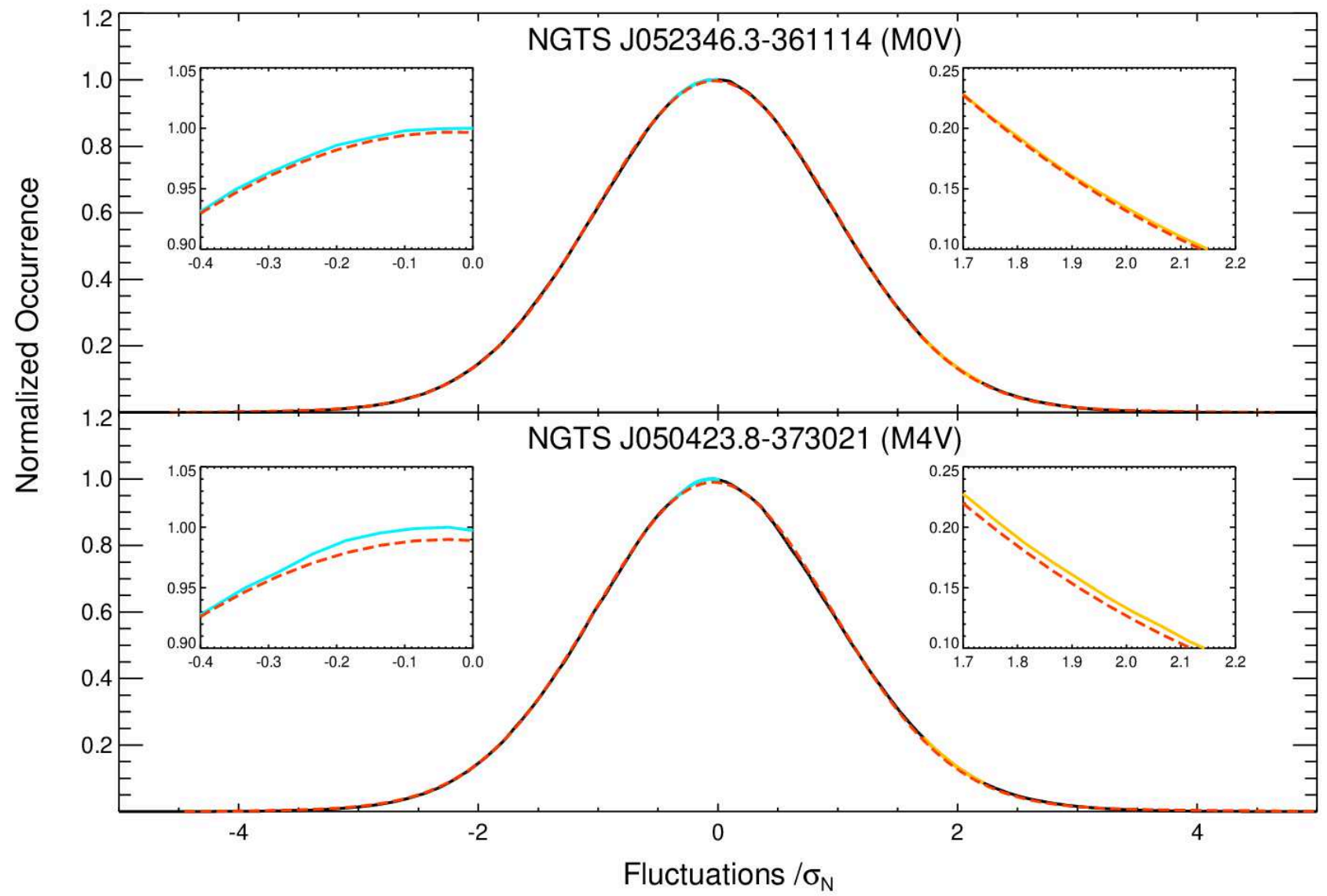

Figure 1. Histograms of intensity fluctuations, each normalised by their respective standard deviations, $\sigma_{N}$, for the NGTS J052346.3-361114 (M0V-type; top panel), and NGTS J050423.8-373021 (M4V-type; lower panel) lightcurves. A standardised Gaussian profile is overplotted in each panel using a dashed red line for reference. The M4V-type distribution has a negative median offset with respect to the Gaussian, in addition to elevated occurrences at $\sim 2 \sigma_{N}$, which is consistent with the statistical signatures of nanoflare activity. On the other hand, the MOV-type intensity fluctuations provide effectively zero negative median offset, and no elevated occurrences at $\sim 2 \sigma_{N}$. This is inconsistent with clear statistical signatures of nanoflare activity, with the resulting distribution remaining more consistent with the presence of photon-based shot noise. Zoomed insets highlight the ranges spanning $-0.4 \leq \sigma_{N} \leq 0.0$ and $1.7 \leq \sigma_{N} \leq 2.2$, where negative median offsets and occurrence excesses, respectively, are clearly visible for the M4V stellar source. For improved clarity, the blue and gold lines display the corresponding distributions in each zoomed panel.

Figure 2 shows the change in the median offset, kurtosis, Fisher skewness, and FW $\frac{1}{8} \mathrm{M}$-to-FWHM ratio (hereafter, $\zeta$ ) of the resulting histograms, respectively, as a function of spectral sub-type, with the results also tabulated in Table 1. From Figure 2, we find a distinct change in the nanoflare statistical signatures as a function of spectral sub-type, suggesting the convective boundary may play an important role in the generation of efficient nanoflare conditions. We find that M2.5V (and beyond) stars exhibit distinct nanoflare statistical signatures that are consistent with those put forward by previous research ${ }^{8}$. Specifically, the average median offset for the pre-M2.5V stars exhibits a large spread around a weakly offset value (upper panel of Figure 2), while the post-M2.5V stars demonstrate a larger consistent offset magnitude (with less uncertainty) of approximately $-0.05 \sigma_{N}$.

The Fisher skewness value is effectively zero for pre-M2.5V stars (second panel from bottom in Figure 2), suggesting no, or very weak, nanoflare activity. From M2.5V onward, there is a clear increasing trend in the Fisher skewness value of the fluctuation distribution, with the M4V sub-type displaying a Fisher skewness equal to $0.051 \pm 0.014$, providing strong evidence for the presence of nanoflares.

The values of kurtosis (second panel from top in Figure 2) have a clear trend, whereby changes in spectral type from M0V to M4V produce increased levels of statistical kurtosis in the corresponding fluctuation distributions. It appears that the degree of statistical kurtosis is influenced directly by the spectral sub-type, with more fully-convective M4V stars demonstrating heightened levels of kurtosis compared with their M0V counterparts. It must be noted that the M3V sub-type does show a dip 


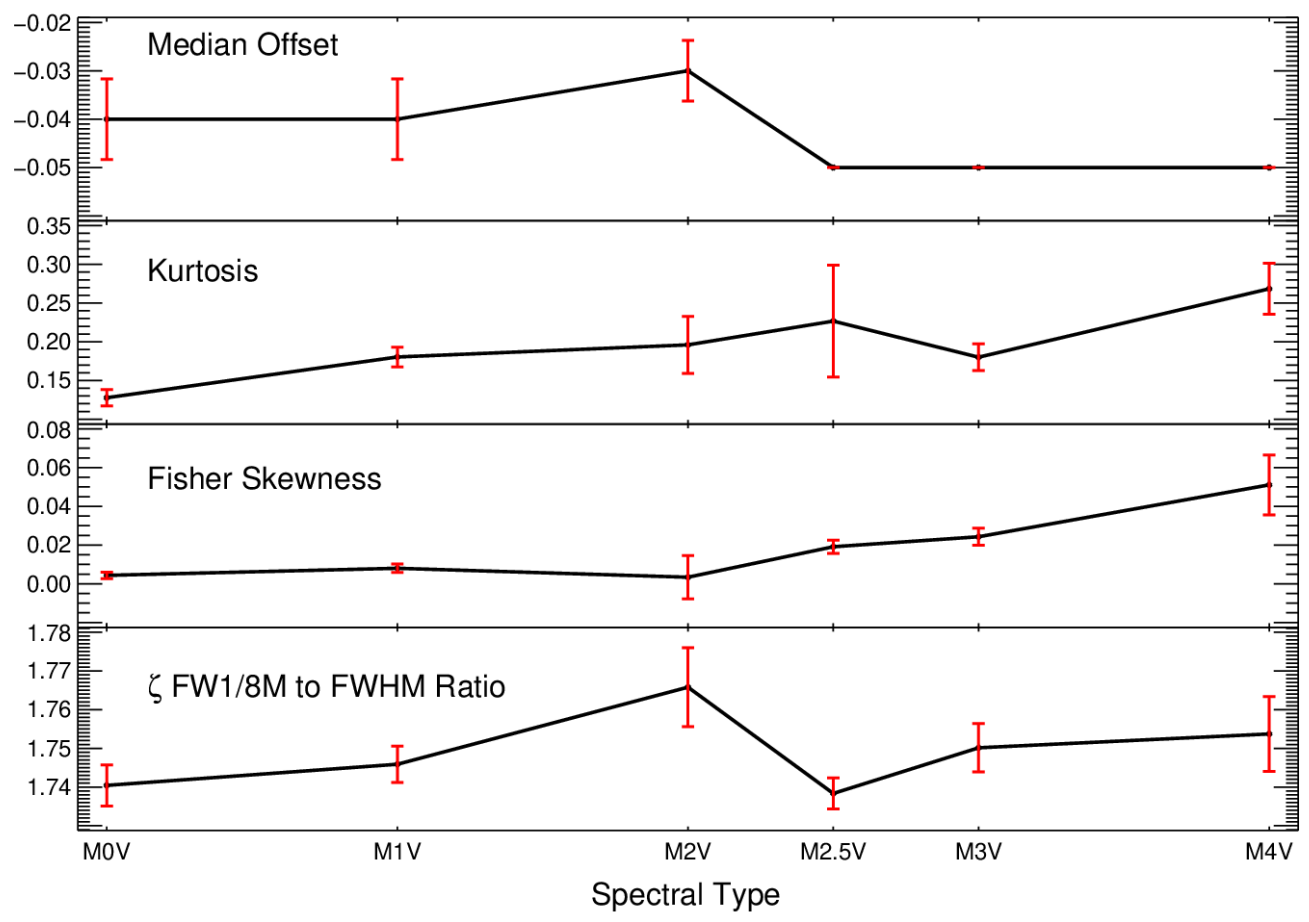

Figure 2. The bootstrap-averaged statistical properties of the intensity fluctuation histograms for each stellar classification. Beyond the convective boundary, at approximately M2.5V and later, sub-types begin to exhibit statistical signatures that are consistent with the presence of nanoflare activity, including larger median offsets (top panel), increasing levels of kurtosis (second panel from top), and higher Fisher skewness values (second panel from bottom). The $\zeta$ (FW $\frac{1}{8} \mathrm{M}$-to-FWHM ratio) values do not vary significantly as a function of stellar classification. However, this is likely due to the interplay between the power-law index of the nanoflares and the duration of the $e$-folding timescales, which are able to counteract the statistical effects of one another.

in its associated kurtosis value, which cannot presently be explained.

There is no clear trend visible in the corresponding $\zeta$ values (lower panel of Figure 2 ) as a function of spectral sub-type. It must be remembered that the $\zeta$ value is a measure of the deviation away from a standard Gaussian distribution, which has a value of $\zeta=1.73$. Increased nanoflare decay timescales (i.e., larger $\tau$ values) result in broader tails of the intensity fluctuation distributions $^{14}$, hence giving rise to $\zeta>1.73$. On the contrary, large power-law indices help reduce the widths of the tails in the intensity fluctuation distributions due to the superposition of positive intensity fluctuations (e.g., new nanoflares) superimposed on top of decaying (i.e., negative) intensity fluctuations, which result in $\zeta<1$.73. As such, the interplay between the power-law index and the nanoflare $e$-folding time produces the specific value of $\zeta$ measured. As such, the relatively consistent values of $\zeta$ found across the spectral range M0V - M4V may result from an increased nanoflare rate expected for M4V stars being negated by an increase in the associated decay timescales of the resulting nanoflares, i.e., a larger $\alpha$ term being coupled with longer $\tau$ values.

Comparing the intensity fluctuation statistical measurements (see, e.g., Table 1 and Figure 2) to those produced by Monte Carlo nanoflare simulations ${ }^{8}$, it is possible to estimate both the power-law index, $\alpha$, and the nanoflare decay timescale, $\tau$, by establishing which combination of flare characteristics best matches the observational signatures. For pre-M2.5V stars, it was not possible to establish values for the power-law index and $e$-folding time that were self-consistent with the Monte Carlo models. For example, it was possible to find self-similarity between the observational and model power-law indices, but this resulted in decay timescales that were incompatible and inconsistent. As a result, we are unable to define nanoflare characteristics for pre-M2.5V stars, suggesting that nanoflare activity may be very weak (or not present) on these specific stellar sub-types. This strongly supports the intensity fluctuation distributions (see, e.g., the upper panel of Figure 1) where the fluctuations closely resemble shot noise patterns (i.e., absent of clear statistical nanoflare signatures).

The statistical parameters for the M2.5V, M3V and M4V stars, which are believed to be beyond the convective boundary and therefore best described as 'fully convective', exhibit values consistent with the power-law indices of $\alpha=2.25 \pm 0.25$ or $\alpha=3.00 \pm 0.25, \alpha=2.25 \pm 0.20$ or $\alpha=3.00 \pm 0.20$, and $\alpha=2.30 \pm 0.20$ or $\alpha=3.10 \pm 0.20$, alongside the $e$-folding timescales of $\tau=200 \pm 100 \mathrm{~s}, \tau=200 \pm 100 \mathrm{~s}$, and $\tau=450 \pm 50 \mathrm{~s}$, respectively (See Table $2_{a}$ ). The approximate symmetry 
(a) Nanoflare parameters per spectral type, derived from statistical properties of Monte-Carlo modelled nanoflare timeseries. The approximately symmetrical distribution of statistical properties leads to an ambiguity in the derived power-law indices, hence $\alpha_{1}$ and $\alpha_{2}$.

\begin{tabular}{|l|l|l|l|}
\hline Spectral Type & $\alpha_{1}$ & $\alpha_{2}$ & $\tau(s)$ \\
\hline M2.5V & $2.25 \pm 0.25$ & $3.00 \pm 0.25$ & $200 \pm 100$ \\
\hline M3V & $2.25 \pm 0.20$ & $3.00 \pm 0.20$ & $200 \pm 100$ \\
\hline M4V & $2.30 \pm 0.20$ & $3.10 \pm 0.20$ & $450 \pm 50$ \\
\hline
\end{tabular}

(b) Nanoflare parameters per spectral type, derived from Fourier properties of Monte-Carlo modelled nanoflare timeseries. There is no ambiguity in the derived power-law indices.

\begin{tabular}{|l|l|l|}
\hline Spectral Type & $\alpha$ & $\tau(s)$ \\
\hline M2.5V & $3.00 \pm 0.15$ & $200 \pm 100$ \\
\hline M3V & $3.00 \pm 0.15$ & $250 \pm 100$ \\
\hline M4V & $3.10 \pm 0.15$ & $450 \pm 50$ \\
\hline
\end{tabular}

Table 2. Nanoflare parameters per spectral type, derived from statistical and Fourier properties of Monte-Carlo modelled nanoflare timeseries respectively.

of the statistical distributions about their peak values leads to ambiguity in the derived power-law indices. As a result, it is possible to map each sub-type onto two distinct solutions for the power-law index. Irrespective of this ambiguity, both sets of possible nanoflare conditions are highly active (i.e., $\alpha>2$ ), in stark contrast to the effectively zero statistical nanoflare signals observed in the pre-M2.5V spectral sub-types. The increased uncertainties in the $\mathrm{M} 2.5 \mathrm{~V}$ power-law indices are due to the larger errors associated with the kurtosis values for these spectral sub-types. M2.5V stars are at the boundary of predicted full convection, so a larger spread in their nanoflare properties would be expected if full convection is the cause of the spectral 'break' in associated power-law indices.

Interestingly, the M4V stars display evidence for longer $e$-folding timescales and larger power-law indices when compared to their $\mathrm{M} 2.5 \mathrm{~V}$ and $\mathrm{M} 3 \mathrm{~V}$ counterparts. As discussed above, this is likely an explanation for the relatively constant $\zeta$ values throughout the spectral sub-type range, whereby the statistical effects of higher power-law indices are negated by the slower decay timescales associated with those stars, which is consistent with previous solar and stellar case studies ${ }^{8,14}$.

Overall, the changes in the statistical parameters indicate that post-convective boundary M-dwarf stars (i.e., M2.5V and later) exhibit greatly enhanced stellar nanoflare activity when compared to the pre-convective boundary M-dwarfs that show little-to-no evidence for nanoflare activity.

\section{Fourier Analysis}

As with the statistical signatures shown in Figure 2, there are dramatic differences in the Fourier properties between M0V and M4V stars. As seen in the Power Spectral Density (PSD) plot of Figure 3, the M0V has an effectively flat power spectrum suggesting no nanoflare signal is present ${ }^{8}$ (i.e., remaining consistent with a shot noise distribution of intensity signals), which is contrasted by the M4V star that demonstrates a spectral slope of $\beta=-0.57 \pm 0.05$ between the frequencies $\sim 0.3-6.0 \mathrm{mHz}$. In Figure 3, the black crosses represent the individual frequency-dependent power measurements, while the solid red line depicts a trendline established over \pm 6 frequency elements $( \pm 0.239 \mathrm{mHz})$. In the lower panel of Figure 3 , a PSD slope is consistent with enhanced rates of stellar nanoflare activity, which begins at the 'turning point' of $0.32 \pm 0.04 \mathrm{mHz}$. The turning point is defined as the initial Fourier peak before the gradual reduction in spectral power with increasing frequency ${ }^{8}$. It must be noted that both PSD plots shown in Figure 3 (i.e., for M0V and M4V spectral types) exhibit numerous power peaks in the range of $1-10 \mathrm{mHz}$, consistent with both stellar nanoflare signatures ${ }^{8}$ and the presence of $p$-mode oscillations generated in the convective layers of M-dwarf stellar sources, where M-dwarf stars are believed to exhibit solar-like oscillations, hence producing $p$-mode signatures synonymous with the typical solar frequency range ${ }^{20,21}$. As the entire range of spectral types included in this study (M0V - M4V) are expected to exhibit $p$-mode oscillations, the peak frequencies within this interval are not conclusive evidence alone of nanoflare activity.

The averaged (following bootstrap procedures) Fourier properties per spectral type are shown in Figure 4, and tabulated in Table 3. As with the averaged statistical signatures shown in Figure 2, there is a marked change in Fourier features consistent with nanoflare activity for spectral classifications M2.5V and later. Evidence for this is shown in the averaged PSD spectral gradient (lower panel of Figure 4), where pre-M2.5V stars have relatively flat spectral slopes $(\beta \sim 0$ ), yet stellar sources beyond the convective boundary at $\mathrm{M} 2.5 \mathrm{~V}$ demonstrate increased magnitude spectral slopes in the range of $-0.6 \leq \beta \leq-0.3$. Note that the peak frequency values (upper panel of Figure 4) are relatively consistent across all M-dwarf stellar sources, approximately in the range of $2-4 \mathrm{mHz}$. As discussed above, this alone does not constitute evidence of nanoflare activity since all of these sources are expected to demonstrate $p$-mode oscillations spanning that particular frequency interval ${ }^{20,22}$.

The corresponding 'turning point', where the spectral slopes are observed to begin, is, of course, equal to zero for the pre-M2.5V stars since they do not exhibit any associated spectral slopes (middle panel of Figure 4). However, for spectral classifications beyond $\mathrm{M} 2.5 \mathrm{~V}$, where the stars are believed to be fully convective, a relatively constant value (when errors are included) in the range of $0.3 \leq f \leq 0.9 \mathrm{mHz}$ is found, which is consistent with previous nanoflare studies ${ }^{8}$. In simulated 


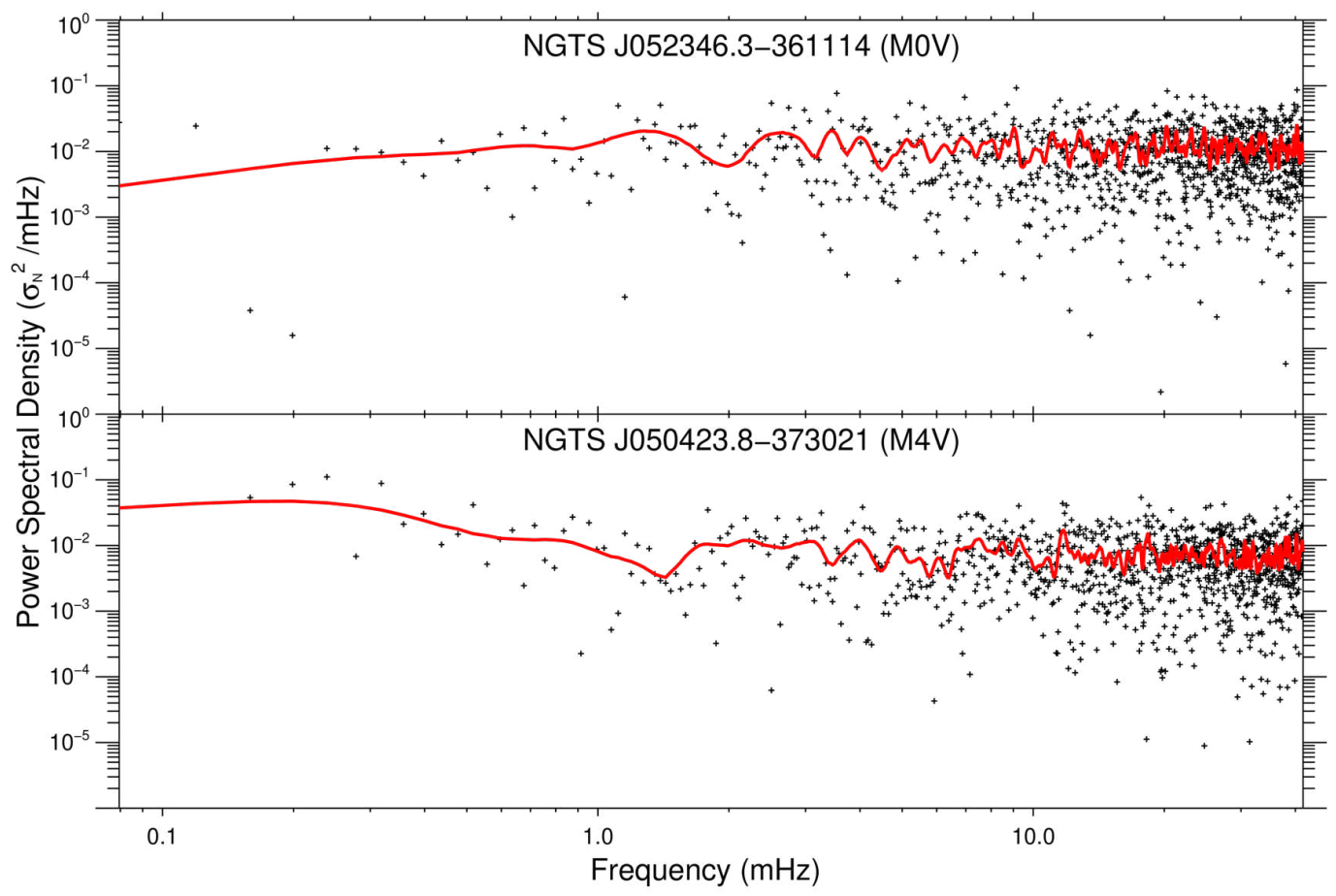

Figure 3. The Fourier power spectral densities (PSDs) for example M0V (upper panel) and M4V (lower panel) stellar sources, displayed in normalised units of $\sigma_{N}^{2} / \mathrm{mHz}$. The crosses in each panel depict the individual power values as a function of frequency, while the solid red line reveals a trendline calculated over \pm 6 frequency elements $( \pm 0.239 \mathrm{mHz})$. It can be seen that the PSD for the MOV star is relatively flat, with small-amplitude power enhancements in the range $3-10 \mathrm{mHz}$, which is consistent with typical $p$-mode oscillations. On the contrary, the PSD for the M4V star exhibits a clear enhancement of spectral energy at lower frequencies, resulting in a spectral slope of $\beta=-0.57 \pm 0.05$ that begins at $0.32 \pm 0.04 \mathrm{mHz}$, followed by numerous power peaks in the range of $1-10 \mathrm{mHz}$, which is consistent with the presence of both nanoflare activity and $p$-mode oscillations.

\begin{tabular}{|l|l|l|l|}
\hline Spectral Type & Gradient & Turning Point $(\mathrm{mHz})$ & Peak frequency $(\mathrm{mHz})$ \\
\hline M0V & $+0.044 \pm 0.023$ & $0.000 \pm 0.000$ & $3.952 \pm 0.320$ \\
\hline M1V & $-0.051 \pm 0.019$ & $0.000 \pm 0.000$ & $2.695 \pm 0.278$ \\
\hline M2V & $-0.035 \pm 0.022$ & $0.000 \pm 0.000$ & $1.956 \pm 0.611$ \\
\hline M2.5V & $-0.326 \pm 0.044$ & $0.762 \pm 0.105$ & $1.821 \pm 0.338$ \\
\hline M3V & $-0.330 \pm 0.022$ & $0.684 \pm 0.063$ & $3.276 \pm 0.557$ \\
\hline M4V & $-0.518 \pm 0.018$ & $0.467 \pm 0.103$ & $1.757 \pm 0.822$ \\
\hline
\end{tabular}

Table 3. Average characteristics of the Fourier PSD properties by each spectral type.

nanoflare lightcurves, an increased flare decay rate (i.e., a longer $\tau$ value) gives rise to a decrease in frequency of the Fourier turning point. Examination of the middle panel of Figure 4 shows that while the turning point frequencies are distinctly different from the pre-M2.5V stars, there does seem to be tentative evidence that the average turning point frequency decreases across the $\mathrm{M} 2.5 \mathrm{~V}, \mathrm{M} 3 \mathrm{~V}$, and M4V spectral types. This is further evidenced in Table 3, where the turning points of the M2.5V, $\mathrm{M} 3 \mathrm{~V}$, and M4V stars are computed as $0.762 \pm 0.105 \mathrm{mHz}, 0.684 \pm 0.063 \mathrm{mHz}$, and $0.467 \pm 0.103 \mathrm{mHz}$, respectively. The evidence suggests that the $e$-folding timescales associated with the M4V stars are longer than their $\mathrm{M} 2.5 \mathrm{~V}$ counterparts, which is consistent with the intensity fluctuation statistical signatures discussed above.

Comparing the derived Fourier properties to the heat maps of simulated signatures shown in Figure 5, it is possible to estimate the power-law indices and decay timescales for each of the M2.5V, M3V, and M4V stellar types that show clear evidence for nanoflare activity. We find power-law indices of $\alpha=3.00 \pm 0.15, \alpha=3.00 \pm 0.15$, and $\alpha=3.10 \pm 0.15$, alongside nanoflare $e$-folding timescales of $\tau=200 \pm 100 \mathrm{~s}, \tau=250 \pm 100 \mathrm{~s}$, and $\tau=450 \pm 50 \mathrm{~s}$, for the M2.5V, M3V, and M4V spectral types, respectively (see Table $22_{b}$ ). Importantly, these values are consistent with the statistical analyses, with the Fourier 


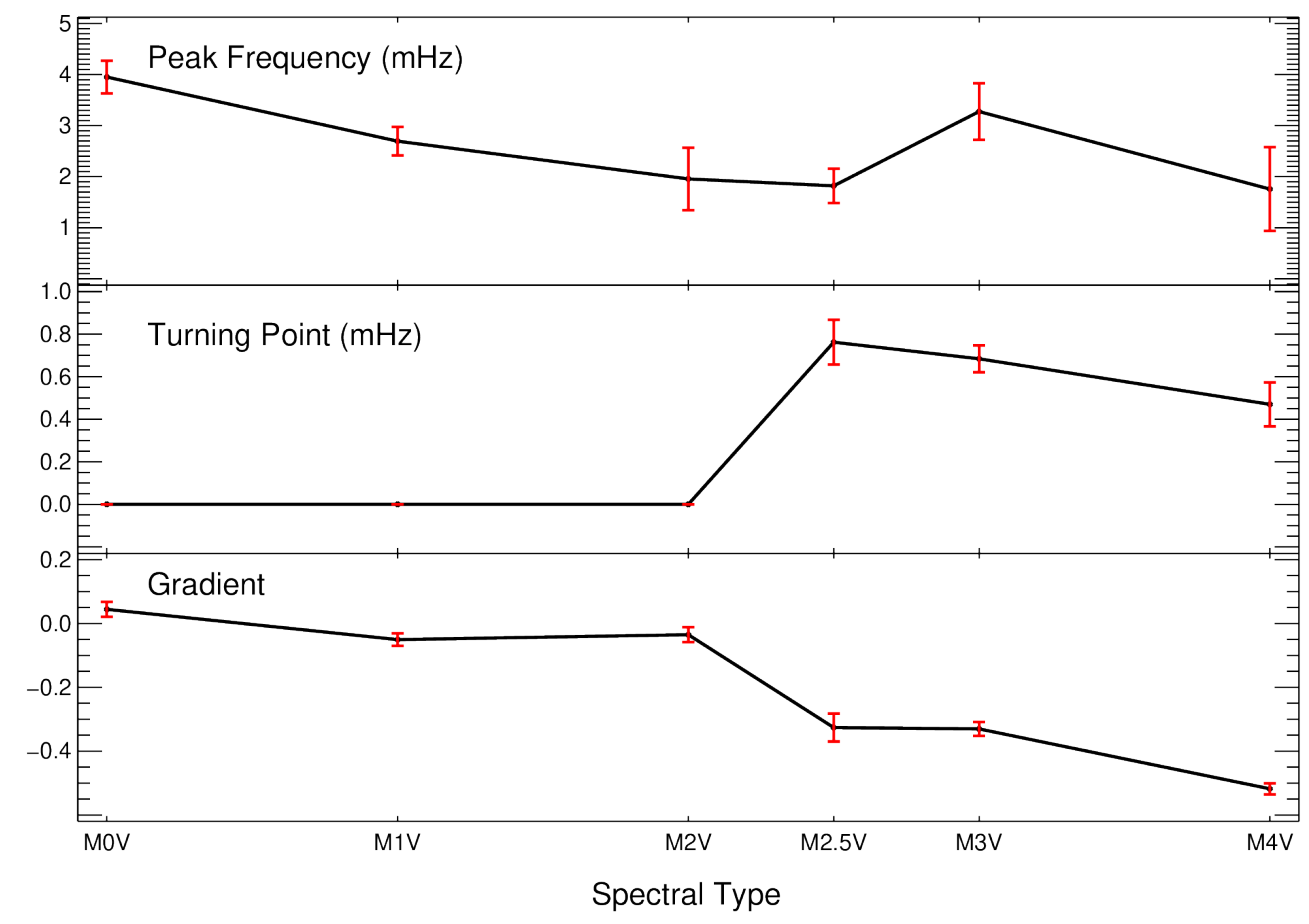

Figure 4. The bootstrap-averaged properties of the Fourier power spectral densities (PSDs) across each spectral type. The upper panel displays the peak frequency values (in $\mathrm{mHz}$ ), which are found to reside within the range of approximately $1-4 \mathrm{mHz}$, which is consistent with both nanoflare activity and $p$-mode oscillations, and therefore cannot be used as an indicator of nanoflare activity by itself. The middle and lower panels display the turning point frequencies (in $\mathrm{mHz}$ ) and subsequent spectral slopes, respectively, as a function of stellar classification. When compared to the Monte Carlo nanoflare simulation outputs depicted in Figure 5, the distinct jump in turning point frequency and spectral gradient at the convective boundary $(\mathrm{M} 2.5 \mathrm{~V}$ ) provides clear evidence of prominent nanoflare activity in $\mathrm{M} 2.5 \mathrm{~V}-\mathrm{M} 4 \mathrm{~V}$ stellar sources.

techniques providing additional benchmarks to validate the nanoflare properties extracted from the observational time series and resolve the ambiguity in power-law index arising from the statistical analysis. In contrast to the statistical mapping, the derived Fourier parameters of the M3V stars are consistent with a marginal $e$-folding time enhancement compared to the M2.5V classifications. This is likely related to the same physical processes that caused enhanced $e$-folding timescales in the M4V stars. However, this is difficult to ascertain due to the relatively large errors in determining the average plasma decay rates over the entire stellar surface.

\section{Evidence of Enhanced Nanoflare Activity Rates in Fully Convective Stars}

Combining the Fourier and statistical analyses, we find that the fully convective M2.5V and M3V sub-types exhibit nanoflare power-law indices of $\alpha=3.00 \pm 0.20$ and $\alpha=3.00 \pm 0.18$, respectively. The M2.5V sub-types are consistent with a decay timescale of $\tau=200 \pm 100 \mathrm{~s}$, whereas the M3V stars display tentative evidence for a slightly enhanced $e$-folding timescale of $\tau=225 \pm 100 \mathrm{~s}$ (these values of $\alpha$ and $\tau$ derived from the combined statistical and Fourier analysis are tabulated in Table 4). These $e$-folding timescales and power-law indices are values consistent with similar M-dwarf spectral types ${ }^{8}$. The M4V stars exhibit elevated power-law indices of $\alpha=3.10 \pm 0.18$, with an increased decay timescale of $\tau=450 \pm 50 \mathrm{~s}$, which we believe may be related to Sweet-Parker reconnection processes.

Convection is a primary driver of magnetic reconnection in stars ${ }^{23}$. As magnetic reconnection is the driving force behind flares, changes to the convective nature of a star have important implications for the resulting flare dynamics. Fully convective stars lack a tachocline, where the radiative interior of the star meets the convective exterior ${ }^{24}$. The tachocline is believed to be responsible for the magnetic dynamo, hence driving magnetic reconnection processes in solar-like stars ${ }^{25}$. In stars with a fully convective atmosphere, the dynamo is theorised to be driven by helical turbulence ${ }^{26-28}$, but this change in dynamo is still under debate. Indeed, investigation into the relationship between stellar rotation and activity levels for fully-convective late M-type dwarf stars have found that the rotation/activity relationship for fully convective stars was almost indistinguishable from partially convective stars, suggesting the activity levels of fully convective stars may somehow be driven by a solar-like dynamo $^{29,30}$. As such, an alternative source for the enhancement of small-scale reconnection may be more likely. Small-scale 
flaring has been shown to occur more favourably via Sweet-Parker reconnection ${ }^{31}$, and the rate of Sweet-Parker reconnection is inversely connected to the plasma Lundquist number, leading to enhanced nanoflaring in stars with low plasma Lundquist numbers. Fully convective stars are believed to host plasma with a higher resistivity value ${ }^{32}$, which lowers the associated plasma Lundquist numbers. If nanoflare rates are enhanced in fully convective stars, then investigating whether this is due to the change in dynamo, or down to the plasma resistivity, could answer important questions regarding the dynamo physics in operation in these stars, and consequently the nature of the solar dynamo.

Small-scale pico/nano-scale flares occur more favourably via Sweet-Parker than Petschek reconnection ${ }^{31}$. This would explain a discontinuity in the power-law relationship between nanoflares and their larger scale counterparts, which remain driven by Petschek-like reconnection ${ }^{33}$. The Sweet-Parker reconnection process is inversely proportional to the square root of the plasma Lundquist number, which is itself inversely proportional to the plasma resistivity. As such, Sweet-Parker reconnection is more favourable in poorly conducting plasmas. The increased decay timescale of $\tau=450 \pm 50 \mathrm{~s}$, alongside the associated increased power-law index of $\alpha=3.10 \pm 0.18$, found for the M4V sub-type may be related to increased plasma resistivity, which matches expectations for mid-to-late M-dwarfs ${ }^{32}$. In contrast, pre-convective boundary (M0V - M2V) stars exhibit weak (if any) nanoflare signals, suggesting that fully convective stellar atmospheres lead to a large enhancement of nanoflare activity.

\begin{tabular}{|l|l|l|}
\hline Spectral Type & $\alpha$ & $\tau(s)$ \\
\hline M2.5V & $3.00 \pm 0.20$ & $200 \pm 100$ \\
\hline M3V & $3.00 \pm 0.18$ & $225 \pm 100$ \\
\hline M4V & $3.10 \pm 0.18$ & $450 \pm 50$ \\
\hline
\end{tabular}

Table 4. Nanoflare parameters per spectral type, derived from combined statistical and Fourier properties of Monte-Carlo modelled nanoflare timeseries.

The enhanced small-scale flare rates in fully convective stars holds profound implications for the energy budgets of those stellar sources. The energy output of rapid and continuous nanoflares may be a major component of the overall stellar energy budget, yet are hidden within the noise envelope of the observations and can only be extracted through use of large-scale statistical and Fourier analyses. The question of whether the enhanced flaring visible in post-convective boundary M2.5V - M4V stars is due to the helical dynamo or altered plasma Lundquist conditions in these stars is an avenue to explore in future work. Furthermore, our work reveals evidence that M4V stars are linked to nanoflare events that have inherently longer decay timescales (i.e., larger $\tau$ values) as well as larger power-law indices. Importantly, mid M-dwarf sub-types should have decreased optical depths, alongside increased plasma resistivities, a trend which continues to late M9 sub-types ${ }^{32}$. If the nanoflare $e$-folding times continue to increase with increasing M-dwarf sub-type, it would support the scenario of increased plasma resistivity leading to increased small-scale flaring via Sweet-Parker reconnection. This would appear to support the findings of ${ }^{29,30}$, that the stellar dynamo in these fully convective stars is not distinct from the solar-like dynamo. As a result, it is of paramount importance to source sufficient late M-type stellar time series for follow-up analyses.

\section{Discussion}

Evidence for stellar nanoflares has been observed on a further 15 post-convective boundary (M2.5V, M3V, and M4V classification) stars, with nanoflare power-law indices and $e$-folding times consistent with the enhanced rates of stellar nanoflare activity previously observed ${ }^{8}$. The marked increase in nanoflare activity is coincident with $\mathrm{M} 2.5 \mathrm{~V}$ and later sub-types, suggesting that the change from partial to fully convective atmospheres may be responsible. The post-convective boundary stars exhibit nanoflare rates that are enhanced from those seen at larger energies in other stars and the Sun, with power-law indices found to be in the region of $\alpha=3.00 \pm 0.20$ for M2.5V and M3V sub-types, with slightly larger values of $\alpha=3.10 \pm 0.18$ for M4V sub-types. The decay timescales for M2.5V and M3V stars were found to be on the order of $\tau=200 \pm 100 \mathrm{~s}$, while evidence was presented for increased plasma $e$-folding times of $\tau=450 \pm 50 \mathrm{~s}$ in the M4V stars, suggesting the presence of Sweet-Parker reconnection processes.

On the contrary, pre-convective boundary M-dwarf (M0V, M1V, and M2V classification) stars exhibit little-to-no statistical or Fourier-based nanoflare signals, suggesting full convection is a requirement for enhanced nanoflare activity. Additionally, the underlying reason why fully convective atmospheres lead to enhanced nanoflare activity should be explored, i.e., is this due to an altered dynamo, or due to other plasma changes such as modification of the corresponding Lundquist number? The latter explanation is our leading theory, due to the altered plasma conditions we find, and the emerging evidence for solar-like behaviour in helical dynamos ${ }^{29,30}$. One avenue of exploration would be examining M5V (and later) stellar types, to investigate if there is a continuing trend in the flare decay rate and associated power-law index, which could be linked to increasing plasma resistivity, and thus increased Sweet-Parker reconnection rates. It is likely such observations would need to be coupled to detailed theoretical and modelling efforts using well-developed numerical simulations ${ }^{34-37}$. 
It goes without saying that enhanced small-scale reconnection in fully convective stars may mean that nanoflare activity could be a significant component of their overall energy budget. Large-scale multi-year studies of stellar nanoflare rates in fully convective M-dwarfs would further our understanding of nanoflare behaviour across different activity cycles, which would further shine light on the ubiquity and role nanoflares play in these dynamic host stars. This can be achieved through further use of large-scale sky surveys (like the NGTS) and space-based observations from the likes of the Transiting Exoplanet Survey Satellite (TESS) ${ }^{38}$, alongside targeted campaigns using high-cadence observational platforms, such as HiPERCAM ${ }^{39}$, or multi-band photometry such as the Rapid Eye Mount (REM) telescope ${ }^{40}$ to investigate the nanoflare signature across layers of the Stellar atmosphere.

\section{Methods}

\section{Observations With NGTS}

To remain consistent with established stellar nanoflare analysis ${ }^{8}$, the Next Generation Transit Survey ${ }^{41}$ (NGTS) was utilised to obtain the observations. The initial spectral classification generated by the NGTS pipeline, which utilises Spectral Energy Distribution fitting ${ }^{41}$, was combined with stellar parameters from the TESS Input Catalog Version $8^{42}$, to ensure robust spectral sub-type identification. The magnitude of the stars (spanning $\sim 12-14$ ), were chosen to match the previous stellar nanoflare study $^{8}$, ensuring similar noise statistics. The long timeseries (each in excess of $10^{5}$ frames) and short cadence $(\sim 12 \mathrm{~s})$ available for thousands of M-type stars allow for the accumulation of suitable number statistics necessary for nanoflare analyses.

After accounting for magnitude considerations, avoiding blended sources, and ensuring TIC matching, we were able to find 5 stars for each spectral sub-type, consisting of M0V, M1V, M2V, M2.5V, M3V, and M4V. The stellar properties including GAIA identifiers, RA, and Dec. of these stars can be found in Table 5. Only one suitable M5V star with TIC-derived stellar parameters could be identified, and no sub-types later than this were found. The intrinsic brightness of M-dwarfs decreases with increasing sub-type ${ }^{43}$, leading to difficulty in identifying suitable candidate stars with the desired brightness properties. Future investigations of post-M4V stars may be fruitful, but identifying a suitable number of candidates may prove difficult with existing instrumentation. Hence, we limit our current study within the range of M0V - M4V, where we have multiple candidates available for comparison. This range also overlaps well with the predicted convective boundary of M2.1-2.3 $\mathrm{V}^{15}$, making it suitable for the study of the role convection plays in the resulting nanoflare activity.

The lightcurves were background corrected and flat-fielded via the NGTS data reduction pipeline described in ${ }^{41}$. This pipeline calculates a relative error in the flux at each data point in the time series. This error correlates with cloudy weather and/or high airmass values. Any fluctuations in this error exceeding $1 \sigma$ above the mean value were removed, resulting in $\sim 10 \%$ of each time series being omitted. This removed any data that had statistically significant increases in its associated flux uncertainties, therefore preventing any large flux errors (largely due to poor seeing conditions) from contaminating the final time series.

Next, the lightcurves extracted for each observing sequence were examined for the presence of macroscopic flare signatures following the same methodology described $\mathrm{by}^{8}$. Specifically, to isolate and remove the macroscopic flares, each lightcurve was searched for emission signatures exceeding $3 \sigma$ above the mean value, lasting continually for a minimum of 1 minute ( 5 datapoints). Based on a normal distribution, the probability of this occurring by chance is $\lesssim 2 \times 10^{-13}$, and hence allowed for the robust detection of intensity fluctuations resulting from macroscopic flaring activity. Every star, apart from the M2V candidate NGTS J062005.7-372555, demonstrated macroscopic flare signatures, resulting in the removal of a further $\sim 0.2-2.5 \%$ of the remaining M-type time series. The degree of macroscopic flare emission varied with the spectral sub-type, with M4V stars exhibiting approximately five times more detected flares than the M0V stellar types. Such bias towards the spectral sub-type is consistent with the studies of ${ }^{44}$ and $^{43}$, where later MV sub-types were also found to exhibit increased macroscopic flare activity. Previous examinations of (macroscopic) stellar flares on late-type MV stars have found power-law indices in the range of $1.5 \leq \alpha \leq 2.7^{11,44-51}$, which are significantly below the nanoflare power-law index $(\alpha \approx 3.25)$ found in similar stars by ${ }^{8}$. As a result, it appears unclear whether a spectral 'break' in the power-law index occurs between large-scale flares and their small-scale counterparts in late-type MV stellar sources, similar to what has been observed in previous studies of solar flares where the power-law index, $\alpha$, does not retain a constant value across all energy scales ${ }^{52-54}$. Hence, it is important to examine the power-law indices associated with nanoflare activity across a wide range of MV spectral sub-types in order to better understand the role convection plays in the generation of nanoflares.

Once the larger-scale flare signatures had been identified, they were subsequently removed from the time series using an interval of \pm 5 minutes ( \pm 25 datapoints) from the first and last detection above the $3 \sigma$ threshold. The number of macroscopic flares removed were used to calculate approximate flare rates for the $\mathrm{M}$ stars. The lightcurves were then normalised on a night by night basis, by the subtraction of a linear line of best fit. To ensure these MV stars had similar activity levels, their stellar rotations periods were examined. The stellar rotation period can be estimated with Lomb-Scargle periodograms ${ }^{55,56}$ This utilises a Fourier transform to calculate the relative power of periodic signatures in a timeseries. A normalised power of 1.0 would indicate a fully periodic signal, whereas 0.0 would be a signal with no periodic nature. In practice, any signal 
greater than 0.1 is a possible periodic signature. Periods in the range 1-30 days were searched for, using a 'frequency-grid' of 20,000 evenly spaced candidate periods (following the procedure utilised by ${ }^{56}$ to identify periods in NGTS stellar timeseries). Common observation aliases such as the day/night cycle, and the lunar cycle were ignored, along with the harmonics of those periods. The majority of the stars did not exhibit candidate rotation periods less than 30 days, indicating they belong to the same population of slow-rotating stars, and hence had similar activity properties. Only the M2V star NGTS J045136.3-321720 deviated from this, with a possible rotation period of $\sim 21$ days. While the associated macroscopic flare activity was elevated with respect to the other partially convective stars (see Table 5), this star still exhibited lower activity than any fully-convective star. Additionally, it exhibited no associated nanoflare activity. As such, this possible increased rotation rate was deemed acceptable.

To ensure consistency with previous stellar nanoflare investigations, the filtering steps employed were identical to those used in $^{8}$, with the filtered lightcurves subsequently cropped to 97060 datapoints each to match the number statistics from the previous study. This allows a direct comparison to be made with the work of $^{8}$, since the previously published nanoflare simulations could be re-used due to identical number statistics, filtering techniques, desired $\alpha$ (power-law index) and $\tau$ (e-folding time) ranges, in addition to specific NGTS-modelled noise characteristics.

\section{Statistical and Fourier Analysis}

Statistical and Fourier analysis were applied to the lightcurves following the normalisation described above, i.e., following flare removal, and detrending. This ensured the input lightcurve is comprised solely of shot and readout noise ${ }^{57,58}$, alongside the background small-scale (nanoflare) stellar activity. These techniques have previously been 'null-tested' 8 ensuring that identically processed A-type and low activity K-type stars do not exhibit the same statistical and Fourier signatures associated with nanoflare activity. This ensured that these statistical and Fourier signatures of nanoflare activity were not a result of the processing itself.

The statistical analyses examines intensity fluctuations in the seemingly quiescent time series following traditional Z-scores approaches ${ }^{59}$. To obtain the intensity fluctuations, each lightcurve is first detrended by a low-order polynomial so the mean value is zero. Then the time series is subsequently renormalized by its own standard deviation, $\sigma_{N}$. Next, a histogram of the fluctuations is generated, with deviations away from a standardised Gaussian distribution identified and compared with those associated with known nanoflare activity.

Flares are found to exhibit two distinct statistical signatures $8,14,60,61$. First is a negative median offset, whereby the median value of the histogram is $<0 \sigma_{N}$, i.e., offset from the mean of the distribution that is equal to $0 \sigma_{N}$ following the polynomial detrending. This is a characteristic signal associated with an exponentially decaying lightcurve, i.e., the decay phase following an impulsive deposition of energy occurs over a longer timescale, hence providing more negative fluctuations that are beneath the elevated signal mean caused by the impulsive event itself. The second nanoflare signature is an excess of fluctuations at $\sim 2 \sigma_{N}$, which is caused by the impulsive nature of the nanoflare energy release. This gives rise to an asymmetric distribution with an excess of fluctuations visible at $\sim 2 \sigma_{N}$ in the corresponding histogram, which can be further benchmarked using Fisher skewness coefficients.

Therefore, a seemingly quiescent lightcurve exhibiting both of these statistical signals is a strong candidate to contain embedded nanoflare signatures. If no nanoflare signal was present, the embedded noise fluctuations would follow a standard Gaussian distribution as a result of Poisson statistics tending to a Gaussian in the limit of large number statistics ${ }^{60}$. In the case of very weak nanoflare activity, only a negative median offset would be present, since the weak impulsive signatures are too faint to provide a fluctuation excess at $\sim 2 \sigma_{N}$ in the corresponding distribution. In addition to the primary nanoflare indicators described above, we also provide benchmarks on the shapes and widths of the intensity fluctuation distributions through calculation of the kurtosis and $\zeta$ values, where $\zeta$ is the ratio of the full-width at eighth-maximum to that of the full-width at half-maximum (i.e., FW $\frac{1}{8}$ M-to-FWHM ratio) of the resulting distribution. Note that a Gaussian distribution will have $\zeta=1.73$, hence deviations from this provide an indication of the intensity fluctuation occurrences taking place close to, and far away from the time series mean.

Following on from the statistical analyses, ${ }^{8}$ employed Fourier techniques to identify the quasi-periodicities of stellar nanoflares, which allows for further paramaterization of the embedded nanoflare activity levels. As highlighted previously ${ }^{8}$, the examination of Fourier signatures, which are derived directly from the stellar lightcurves, can help disambiguate any derived nanoflare characteristics and further substantiate the evidence for specific activity levels. Power spectral densities (PSDs) were derived from the stellar time series using conventional methods ${ }^{62,63}$. A trendline was established over \pm 6 frequency elements $( \pm 0.239 \mathrm{mHz})$. This revealed power peaks in the same frequency range $(\sim 1-10 \mathrm{mHz})$ as previously interpreted $p$-mode signatures ${ }^{20,21,64}$, despite being a consequence of embedded nanoflare activity. Additionally, the PSDs displayed a prominent spectral slope following the peak energy value, which was also found to be due to the underlying nanoflare signal. There was an associated turning point, the frequency at which the spectral slope began to become evident, and an initial power-peak was found. These features were a further characteristic nanoflare signal. 
The statistical and Fourier signatures were reported for each star. These properties were averaged per spectral type, by using a bootstrap average. The IDL package bootstrap_mean.pro was used, with 1000 bootstrap resamples, and a confidence interval of 0.68 .

\section{Monte-Carlo Nanoflare Simulation}

To substantiate the observational findings, simulated nanoflare time series were subjected to the same statistical and Fourier analyses. The 1-D simulations consisted of artificial Poisson background noise (tuned to match the observational noise properties of the NGTS) alongside simulated nanoflare signals. The simulated nanoflares were generated using a wide spectrum of $\alpha$ (power-law index) and $\tau$ ( $e$-folding time) values, which allowed for a dense grid of possible nanoflare conditions to be assimilated. The input power-law $\alpha$ was varied in steps of 0.05 from $1 \leq \alpha \leq 4$. The $\tau$ value was varied from $5 \leq \tau \leq 500 \mathrm{~s}$ in steps of $5 \mathrm{~s}$. This created an input grid of 6100 possible nanoflare conditions. The resulting simulated time series appeared quiescent and were therefore visually indistinguishable from the observational lightcurves, although they contained embedded nanoflare signatures that could be used to benchmark the level of nanoflare activity in the observations. Each of the 6100 Monte-Carlo noise simulations were matched to the same 97060 datapoints to ensure consistent number statistics with the observational dataset. The same normalisation steps applied to the observational data were applied to the modelled timeseries, i.e., $3 \sigma$ threshold 'flare' subtraction, low order polynomial detrending, and the z-score normalisation. These Monte-Carlo models were first developed for previous stellar nanoflare analysis ${ }^{8}$, and fully described therein. The nanoflare modelling techniques were originally adapted from 2-D Solar nanoflare modelling code, which have been documented extensively ${ }^{14}$.

The nanoflare simulation requires several key input parameters. A simple flare model forms the basis of these simulations. The flare begins with an impulsive energy deposition followed by approximately exponential decay. The $\alpha$ and $\tau$ values will dictate the frequency of energy release, and the time taken for the flare to decay. The modelled flare-decay rate, $\tau$ was varied by $\pm 10 \%$ in each simulated timeseries. These decay rates are a function of the underlying plasma conditions, and hence are not consistent over time. A variation of $\pm 10 \%$ allows for a more realistic representation of the approximately exponential decay timescales. The energy range of nanoflares spanned $10^{22}-10^{25}$ ergs. These flare energies are generated using a random power-law generation function. This will randomly order energy values according to the input power-law index, $\alpha$. These events are then 'decayed' via an exponential decay with the characteristic timescale being within $\pm 10 \%$ of the input $\tau$ value. The power-law distribution only gives information about the relative frequency and energy release of flare events, but does not provide the number of occurrences of these events. As such, the number of flare events needs to be normalised, ensuring a given power-law index leads to the correct flare energy release rate. This normalisation is achieved through comparison with the work of ${ }^{6}$ and $^{65}{ }^{65}$ find that for an energy of $10^{25} \mathrm{ergs}$, the expected flare frequency is $\sim 2000 \times 10^{-50} \mathrm{erg}^{-1} \mathrm{~cm}^{-2} \mathrm{~s}^{-1}$. This allows an approximate lower-limit to the number of flare events to be set. This normalisation requires the underlying stellar area. As these stars are point-sources, the simulated area was the entire Earth-facing area of the star. This was set to $\sim 10^{21} \mathrm{~cm}^{2}$ . This is the approximate surface area of an M-dwarf ${ }^{66}$. While larger flares are reliant on highly localised and intense magnetic conditions, nanoflares are expected to occur continuously throughout the entire stellar atmosphere. As such, the entire stellar surface may be treated as a source of nanoflare activity. Combined with an exposure time and cadence matched to observation (i.e., 10 seconds, and 12 seconds respectively for the NGTS), the number of nanoflare events per second were normalised for each simulation. A direct one-to-one scaling was used to convert the flare energies into 'counts'. The whitelight NGTS observations capture the majority of the flare energy, as flares (particularly M-dwarf flares ${ }^{67}$ ) emit strongly in whitelight and at UV wavelengths ${ }^{68,69}$. The flare energies themselves are assumed to scale linearly with area ${ }^{43}$. As such, we convert our simulated flare energies directly into counts. A ' $1 \sigma$ ' flare energy is used to 'normalise' this conversion. This is an estimate of the flare energy corresponding to a $1 \sigma$ fluctuation. This requires a degree of estimation, and is dependent on the underlying observation platform. This should be set to the approximate energy level of the noise floor, i.e., the energy of an event within the noise floor. A value in the range $10^{24}-10^{25}$ ergs would set the noise floor at the upper span of nanoflaring energies, an appropriate estimate for most observation systems, as these nanoflares remain on or below the noise-floor. The value of $5 \times 10^{24}$ ergs was used as the $1 \sigma$ value in this work. This energy ensures that the span of these nanoflare fluctuations remain within the noise envelope, with a maximum fluctuation of $2.5 \sigma$ at the upper energy of $10^{25} \mathrm{ergs}$. This ensures that the modelled timeseries remain seemingly 'quiescent', matching it to their companion observational timeseries.

The statistical and Fourier signatures of these simulated timeseries were output as 2-D 'heat-maps', allowing direct comparison between observed and simulated statistical and Fourier properties, to diagnose the underlying nanoflare conditions.

While efforts were made to match as many properties to the original study(to ensure consistent number statistics), there was a slight deviation in the Fourier analysis. The longest continuous time series (i.e., the longest uninterrupted series of frames) common to the observed stars in this study was 2095 datapoints, slightly shorter than the 2316 consecutive frames employed by $^{8}$. This resulted in the frequency resolution being slightly reduced from $\Delta f=0.0356 \mathrm{mHz}$ to $\Delta f=0.0398 \mathrm{mHz}$ in the present study. In order to readily compare the observational PSDs to those calculated from the Monte Carlo nanoflare models, the Fourier signatures needed to be re-calculated adhering to the new frequency resolution. Hence, utilising the new frequency 

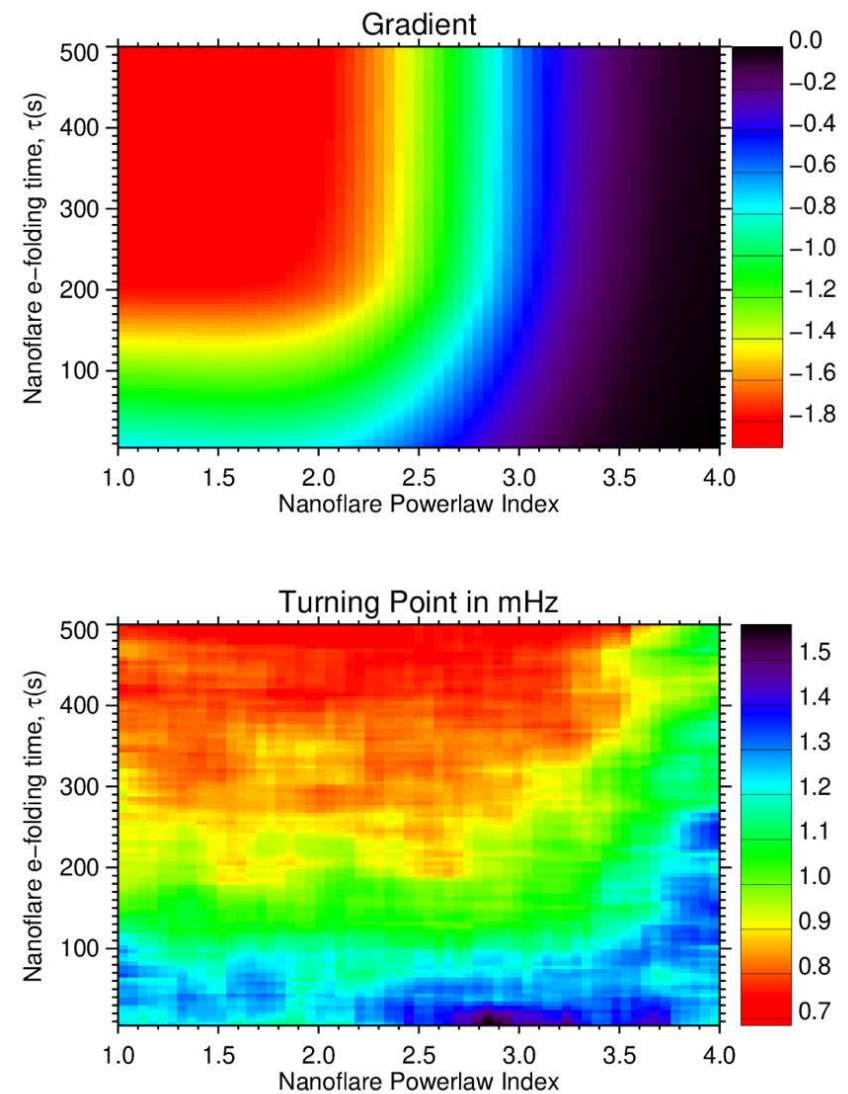
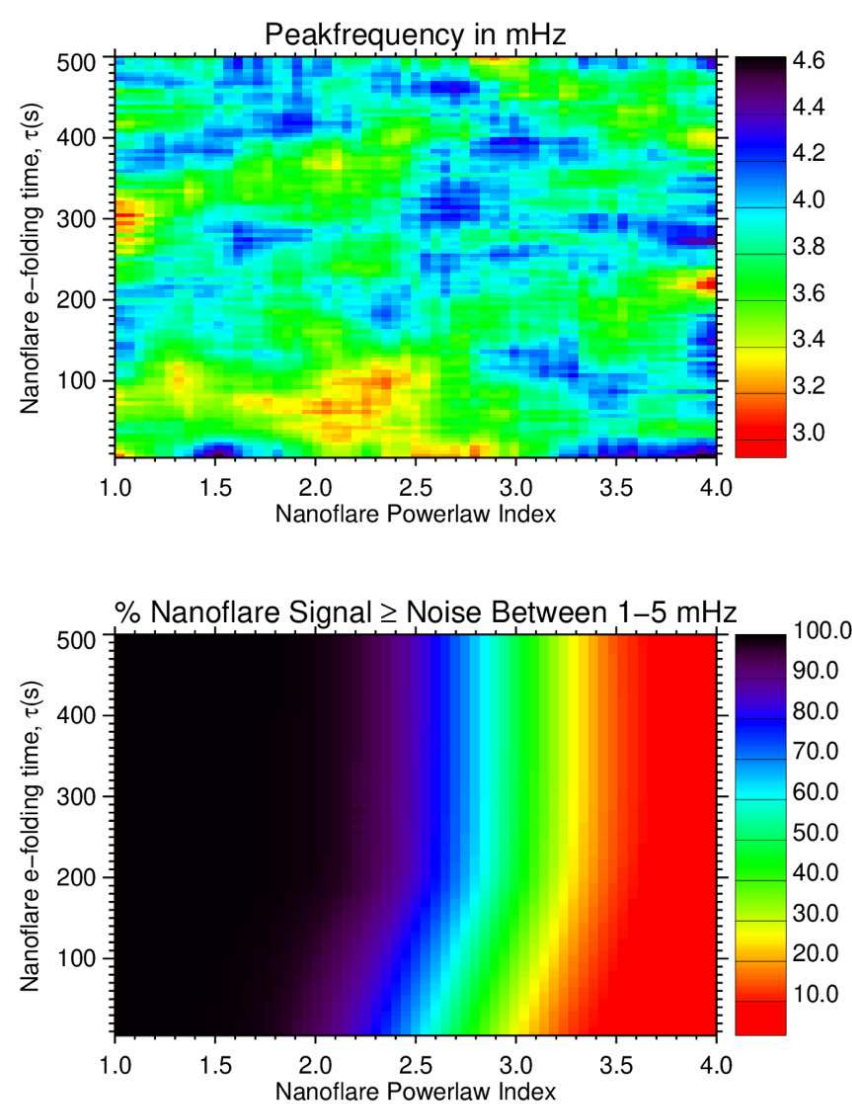

Figure 5. A reproduction of Figure $7 \mathrm{from}^{8}$, with the constituent PSDs re-calculated for 2095 datapoints to match the longest continuous time series used in the present study. The primary peak frequencies (lower-left), spectral slopes (upper-left), dominant frequencies following detrending (upper-right), and the percentage of nanoflare power above the noise floor in the range of $1-5 \mathrm{mHz}$ (lower-right), is displayed as a function of the power-law index, $\alpha$, and the decay timescale, $\tau$, used to generate the synthetic time series. While a few individual values differ, the overall trends and the magnitude of the derived signals are consistent with the PSD properties generated from 2316 data points ${ }^{8}$.

resolution, we re-computed the PSDs and corresponding 'heat map' of the simulated Fourier properties as a function of both the nanoflare power-law and $e$-folding time. The recalculated heat map is displayed in Figure 5. Due to the change in frequency resolution being a relatively small value $(0.0042 \mathrm{mHz})$, no noticeable deviations from Figure 5 and the original distribution can be seen, with the trends identical in both studies. This is likely a result of the Fourier nanoflare trends being relatively broad in frequency with peak values sufficiently away from the lowest and highest (i.e., Nyquist) frequencies of the time series, hence are not significantly affected by very small changes in the underlying frequency resolution.

signals are consistent with the PSD properties generated from 2316 data points.

\section{References}

1. Priest, E. R. Role of magnetic reconnection in solar flares. Adv. Space Res. 6, 73-82, DOI: 10.1016/0273-1177(86)90120-1 (1986).

2. Reale, F. Diagnostics of stellar flares from X-ray observations: from the decay to the rise phase. Astron. Astrophys. 471, 271-279, DOI: 10.1051/0004-6361:20077223 (2007). 0705.3254.

3. Cargill, P. J., Warren, H. P. \& Bradshaw, S. J. Modelling nanoflares in active regions and implications for coronal heating mechanisms. Philos. Transactions Royal Soc. Lond. Ser. A 373, 20140260-20140260, DOI: 10.1098/rsta.2014.0260 (2015).

4. Cargill, P. J. \& Klimchuk, J. A. Nanoflare Heating of the Corona Revisited. Astrophys. J. 605, 911-920, DOI: 10.1086/ 382526 (2004). 
5. Antiochos, S. K. \& Sturrock, P. A. Evaporative cooling of flare plasma. Astrophys. J. 220, 1137-1143, DOI: 10.1086/155999 (1978).

6. Aschwanden, M. J. et al. Time Variability of the "Quiet" Sun Observed with TRACE. II. Physical Parameters, Temperature Evolution, and Energetics of Extreme-Ultraviolet Nanoflares. Astrophys. J. 535, 1047-1065, DOI: 10.1086/308867 (2000).

7. Parker, E. N. Nanoflares and the Solar X-Ray Corona. Astrophys. J. 330, 474, DOI: 10.1086/166485 (1988).

8. Dillon, C. J. et al. Statistical Signatures of Nanoflare Activity. II. A Nanoflare Explanation for Periodic Brightenings in Flare Stars Observed by NGTS. Astrophys. J. 904, 109, DOI: 10.3847/1538-4357/abbfa8 (2020). 2010.04167.

9. Klimchuk, J. A. On solving the coronal heating problem. Sol. Phys. 234, 41-77, DOI: 10.1007/s11207-006-0055-z (2006).

10. Benz, A. O. Flare observations. Living Rev. Sol. Phys. 14, 2, DOI: 10.1007/s41116-016-0004-3 (2016).

11. Hudson, H. S. Solar flares, microflares, nanoflares, and coronal heating. Sol. Phys. 133, 357-369, DOI: 10.1007/ BF00149894 (1991).

12. Aschwanden, M. J. Do EUV Nanoflares Account for Coronal Heating? Sol. Phys. 190, 233-247, DOI: 10.1023/A: 1005288725034 (1999).

13. Aschwanden, M. J., Winebarger, A., Tsiklauri, D. \& Peter, H. The Coronal Heating Paradox. Astrophys. J. 659, 1673-1681, DOI: $10.1086 / 513070(2007)$.

14. Jess, D. B. et al. Statistical Signatures of Nanoflare Activity. I. Monte Carlo Simulations and Parameter-space Exploration. Astrophys. J. 871, 133, DOI: 10.3847/1538-4357/aaf8ae (2019). 1812.06978.

15. Mullan, D. J. \& Houdebine, E. R. A Transition of Dynamo Modes in M Dwarfs: Narrowing Down the Spectral Range Where the Transition Occurs. Astrophys. J. 891, 128, DOI: 10.3847/1538-4357/ab6ffa (2020). 2001.08721.

16. Efron, B. et al. Bootstrap methods: Another look at the jackknife. Annals Stat. 7, 1-26 (1979).

17. Simpson, G. \& Mayer-Hasselwander, H. Bootstrap sampling - Applications in gamma-ray astronomy. Astron. Astrophys. 162, 340-348 (1986).

18. Desmars, J., Arlot, S., Arlot, J. E., Lainey, V. \& Vienne, A. Estimating the accuracy of satellite ephemerides using the bootstrap method. Astron. Astrophys. 499, 321-330, DOI: 10.1051/0004-6361/200811509 (2009).

19. Yao, J. M., Manchester, R. N. \& Wang, N. Determination of the Sun's offset from the Galactic plane using pulsars. Mon. Not. R. Astron. Soc. 468, 3289-3294, DOI: 10.1093/mnras/stx729 (2017). 1704.01272.

20. Rodríguez-López, C., MacDonald, J., Amado, P. J., Moya, A. \& Mullan, D. The theoretical instability strip of M dwarf stars. Mon. Not. R. Astron. Soc. 438, 2371-2379, DOI: 10.1093/mnras/stt2352 (2014). 1312.2743.

21. Rodríguez, E. et al. Search for pulsations in M dwarfs in the Kepler short-cadence data base. Mon. Not. R. Astron. Soc. 457, 1851-1863, DOI: 10.1093/mnras/stw033 (2016).

22. Guenther, D. B. et al. The Nature of p-Modes and Granulation in Procyon: New MOST Photometry and New Yale Convection Models. Astrophys. J. 687, 1448-1459, DOI: 10.1086/592060 (2008).

23. Pedersen, M. G. et al. Do A-type stars flare? Mon. Not. R. Astron. Soc. 466, 3060-3076, DOI: 10.1093/mnras/stw3226 (2017). 1612.04575.

24. Spiegel, E. A. \& Zahn, J. P. The solar tachocline. Astron. Astrophys. 265, 106-114 (1992).

25. Parfrey, K. P. \& Menou, K. The Origin of Solar Activity in the Tachocline. Astrophys. J. Lett. 667, L207-L210, DOI: 10.1086/522426 (2007). 0708.3675.

26. Durney, B. R., De Young, D. S. \& Roxburgh, I. W. On the Generation of the Largescale and Turbulent Magnetic Fields in the Solar Type Stars. Sol. Phys. 145, 207-225, DOI: 10.1007/BF00690652 (1993).

27. Browning, M. K. Simulations of Dynamo Action in Fully Convective Stars. Astrophys. J. 676, 1262-1280, DOI: 10.1086/527432 (2008). 0712.1603.

28. Pipin, V. V. \& Seehafer, N. Stellar dynamos with $\omega \times j$ effect. A@AND@A 493, 819-828, DOI: 10.1051/0004-6361: 200810766 (2009).

29. Wright, N. J. \& Drake, J. J. Solar-type dynamo behaviour in fully convective stars without a tachocline. Nature 535, 526-528, DOI: 10.1038/nature18638 (2016). 1607.07870.

30. Wright, N. J., Newton, E. R., Williams, P. K. G., Drake, J. J. \& Yadav, R. K. The stellar rotation-activity relationship in fully convective M dwarfs. Mon. Not. R. Astron. Soc. 479, 2351-2360, DOI: 10.1093/mnras/sty1670 (2018). 1807.03304. 
31. Tsuneta, S. \& Katsukawa, Y. Coronal Heating with Sweet-Parker Picoflares, vol. 325 of Astronomical Society of the Pacific Conference Series, 289 (2004).

32. Mohanty, S., Basri, G., Shu, F., Allard, F. \& Chabrier, G. Activity in Very Cool Stars: Magnetic Dissipation in Late M and L Dwarf Atmospheres. Astrophys. J. 571, 469-486, DOI: 10.1086/339911 (2002). astro-ph/0201518.

33. Loureiro, N. F. \& Uzdensky, D. A. Magnetic reconnection: from the Sweet-Parker model to stochastic plasmoid chains. Plasma Phys. Control. Fusion 58, 014021, DOI: 10.1088/0741-3335/58/1/014021 (2016). 1507.07756.

34. Takahashi, H. R., Kudoh, T., Masada, Y. \& Matsumoto, J. Scaling Law of Relativistic Sweet-Parker-type Magnetic Reconnection. Astrophys. J. Lett. 739, L53, DOI: 10.1088/2041-8205/739/2/L53 (2011). 1108.3891.

35. Tenerani, A., Velli, M., Rappazzo, A. F. \& Pucci, F. Magnetic Reconnection: Recursive Current Sheet Collapse Triggered by "Ideal" Tearing. Astrophys. J. Lett. 813, L32, DOI: 10.1088/2041-8205/813/2/L32 (2015). 1506.08921.

36. Shi, C., Velli, M. \& Tenerani, A. Marginal Stability of Sweet-Parker Type Current Sheets at Low Lundquist Numbers. Astrophys. J. 859, 83, DOI: 10.3847/1538-4357/aabd83 (2018). 1802.07162.

37. Papini, E., Landi, S. \& Del Zanna, L. Fast Magnetic Reconnection: Secondary Tearing Instability and Role of the Hall Term. Astrophys. J. 885, 56, DOI: 10.3847/1538-4357/ab4352 (2019). 1906.06779.

38. Ricker, G. R. et al. Transiting Exoplanet Survey Satellite (TESS). In Proc. SPIE, vol. 9143 of Society of Photo-Optical Instrumentation Engineers (SPIE) Conference Series, 914320, DOI: 10.1117/12.2063489 (2014). 1406.0151.

39. Dhillon, V. S. et al. HiPERCAM: a high-speed quintuple-beam CCD camera for the study of rapid variability in the universe. In Proc. SPIE, vol. 9908 of Society of Photo-Optical Instrumentation Engineers (SPIE) Conference Series, 99080Y, DOI: 10.1117/12.2229055 (2016). 1606.09214.

40. Antonelli, L. A. The rem telescope: a robotic facility to promptly follow-up grbs and cosmic fast transients. AIP Conf. Proc. 797, 173-180, DOI: 10.1063/1.2130230 (2005). https://aip.scitation.org/doi/pdf/10.1063/1.2130230.

41. Wheatley, P. J. et al. The Next Generation Transit Survey (NGTS). Mon. Not. R. Astron. Soc. 475, 4476-4493, DOI: 10.1093/mnras/stx2836 (2018). 1710.11100.

42. Stassun, K. G. et al. The TESS Input Catalog and Candidate Target List. Astron. J. 156, 102, DOI: 10.3847/1538-3881/ aad050 (2018). 1706.00495.

43. Yang, H. et al. The flaring activity of $\mathrm{m}$ dwarfs in the kepler field. The Astrophys. J. 849, 36, DOI: 10.3847/1538-4357/ aa8ea2 (2017).

44. Hawley, S. L. et al. Kepler Flares. I. Active and Inactive M Dwarfs. Astrophys. J. 797, 121, DOI: 10.1088/0004-637X/797/ 2/121 (2014). 1410.7779.

45. Robinson, R. D., Carpenter, K. G., Percival, J. W. \& Bookbinder, J. A. A Search for Microflaring Activity on dMe Flare Stars. I. Observations of the dM8e Star CN Leonis. Astrophys. J. 451, 795, DOI: 10.1086/176266 (1995).

46. Robinson, R. D., Carpenter, K. G. \& Percival, J. W. A Search for Microflaring Activity on DME Flare Stars. II. Observations of YZ Canis Minoris. Astrophys. J. 516, 916-923, DOI: 10.1086/307133 (1999).

47. Kashyap, V. L., Drake, J. J., Güdel, M. \& Audard, M. Flare Heating in Stellar Coronae. Astrophys. J. 580, 1118-1132, DOI: $10.1086 / 343869$ (2002). astro-ph/0208546.

48. Güdel, M., Audard, M., Kashyap, V. L., Drake, J. J. \& Guinan, E. F. Are coronae of magnetically active stars heated by flares? II. extreme ultraviolet and x-ray flare statistics and the differential emission measure distribution. The Astrophys. $J$. 582, 423-442, DOI: $10.1086 / 344614$ (2003).

49. Güdel, M. X-ray astronomy of stellar coronae. Astron. Astrophys. Rev. 12, 71-237, DOI: 10.1007/s00159-004-0023-2 (2004). astro-ph/0406661.

50. Welsh, B. Y. et al. GALEX high time-resolution ultraviolet observations of dMe flare events. Astron. Astrophys. 458, 921-930, DOI: 10.1051/0004-6361:20065304 (2006). astro-ph/0608254.

51. Reale, F. Plasma Sloshing in Pulse-heated Solar and Stellar Coronal Loops. Astrophys. J. Lett. 826, L20, DOI: 10.3847/ 2041-8205/826/2/L20 (2016). 1607.01329.

52. Wang, F. Y. \& Dai, Z. G. Self-organized criticality in X-ray flares of gamma-ray-burst afterglows. Nat. Phys. 9, 465-467, DOI: 10.1038/nphys2670 (2013). 1308.1253.

53. Ryan, D. F., Dominique, M., Seaton, D., Stegen, K. \& White, A. Effects of flare definitions on the statistics of derived flare distributions. Astron. Astrophys. 592, A133, DOI: 10.1051/0004-6361/201628130 (2016). 1606.04472. 
54. Milligan, R. O., Hudson, H. S., Chamberlin, P. C., Hannah, I. G. \& Hayes, L. A. Lyman-alpha Variability During Solar Flares Over Solar Cycle 24 Using GOES-15/EUVS-E. Space Weather. 18, e02331, DOI: 10.1029/2019SW002331 (2020). 1910.01364.

55. VanderPlas, J. T. Understanding the lomb-scargle periodogram. The Astrophys. J. Suppl. Ser. 236, 16, DOI: 10.3847/ 1538-4365/aab766 (2018).

56. Jackman, J. A. G. et al. Stellar flares detected with the Next Generation Transit Survey. Mon. Not. R. Astron. Soc. 504, 3246-3264, DOI: 10.1093/mnras/stab979 (2021). 2104.02648.

57. Terrell, J. Size limits on fluctuating astronomical sources. Astrophys. J. Lett. 213, L93-L97, DOI: 10.1086/182417 (1977).

58. Delouille, V., Chainais, P. \& Hochedez, J. F. Spatial and Temporal Noise in Solar EUV Observations. Sol. Phys. 248, 441-455, DOI: 10.1007/s11207-008-9131-x (2008).

59. Sprinthall, R. Basic Statistical Analysis (Prentice Hall, 1990).

60. Terzo, S. et al. Widespread Nanoflare Variability Detected with Hinode/X-Ray Telescope in a Solar Active Region. Astrophys. J. 736, 111, DOI: 10.1088/0004-637X/736/2/111 (2011). 1105.2506.

61. Jess, D. B., Mathioudakis, M. \& Keys, P. H. Nanoflare Activity in the Solar Chromosphere. Astrophys. J. 795, 172, DOI: 10.1088/0004-637X/795/2/172 (2014). 1409.6726.

62. Welch, P. D. A direct digital method of power spectrum estimation. IBM J. Res. Dev. 5, 141-156, DOI: 10.1147/rd.52.0141 (1961).

63. Vaughan, S. Random time series in astronomy. Philos. Transactions Royal Soc. Lond. Ser. A 371, 20110549-20110549, DOI: 10.1098/rsta.2011.0549 (2012).

64. Andrews, A. D. Investigation of micro-flaring and secular and quasi-periodic variations in dMe flare stars. I. Suspected ultra-short "waves" in the dM2-3estar V1285 Aquilae. Astron. Astrophys. 210, 303-310 (1989).

65. Parnell, C. E. \& Jupp, P. E. Statistical Analysis of the Energy Distribution of Nanoflares in the Quiet Sun. Astrophys. J. 529, 554-569, DOI: 10.1086/308271 (2000).

66. Reid, I. N. \& Hawley, S. L. New light on dark stars : red dwarfs, low-mass stars, brown dwarfs (Springer-Verlag Berlin Heidelberg, 2005).

67. Walkowicz, L. M. et al. White-light Flares on Cool Stars in the Kepler Quarter 1 Data. Astron. J. 141, 50, DOI: 10.1088/0004-6256/141/2/50 (2011). 1008.0853.

68. Woods, T. N., Kopp, G. \& Chamberlin, P. C. Contributions of the solar ultraviolet irradiance to the total solar irradiance during large flares. J. Geophys. Res. (Space Physics) 111, A10S14, DOI: 10.1029/2005JA011507 (2006).

69. Schmitt, J. H. M. M., Kanbach, G., Rau, A. \& Steinle, H. Optical microflaring on the nearby flare star binary UV Ceti. Astron. Astrophys. 589, A48, DOI: 10.1051/0004-6361/201628199 (2016).

\section{Acknowledgements}

C.J.D., D.B.J. and S.D.T.G. wish to thank Invest NI and Randox Laboratories Ltd. for the award of a Research and Development Grant (059RDEN-1) that allowed the computational techniques employed to be developed. D.B.J. would like to thank the UK Science and Technology Facilities Council (STFC) for an Ernest Rutherford Fellowship (ST/K004220/1), in addition to a dedicated standard grant (ST/L002744/1) that allowed this project to be started. D.B.J. and M.M. would like to thank STFC for the consolidated grant ST/T00021X/1. J.A.G.J. acknowledges support from grant HST-GO-15955.004-A from the Space Telescope Science Institute, which is operated by the Association of Universities for Research in Astronomy, Inc., under NASA contract NAS 5-26555 S.L.C would like to thank STFC for an Ernest Rutherford Fellowship (ST/R003726/1). P.J.W., D.R.A., and R.G.W. acknowledge support from STFC consolidated grants ST/L000733/1 and ST/P000495/1. This project is based on data collected under the NGTS project at the ESO La Silla Paranal Observatory. The NGTS facility is operated by the consortium institutes with support from the UK STFC under projects ST/M001962/1 and ST/S002642/1. D.B.J. wishes to acknowledge scientific discussions with the Waves in the Lower Solar Atmosphere (WaLSA; www.WaLSA.team) team, which is supported by the Research Council of Norway (project no. 262622) and the Royal Society (award no. Hooke18b/SCTM). JIV acknowledges support of CONICYT-PFCHA/Doctorado Nacional-21191829. 


\section{${ }_{548}$ Author contributions statement}

C.J.D, D.B.J, and M.M. conceived the experiment. C.A.W. identified the potential to observe stellar nanoflares through the use of NGTS, as well as benchmarking/quality control science verification checks. C.J.D conducted the experiment and analysed the results. C.J.D and D.B.J prepared the manuscript. C.A.W, J.A.G.J., P.J.W, M.R.G, S.L.C, D.R.A, M.R.B, R.G.W, and J.I.V provided the observational products as part of the NGTS. All authors reviewed the manuscript, and provided input.

\section{Additional information}

Observational data supporting the findings of this study are available from the NGTS 2020 data release, https://archive.eso.org/cms/esoarchive-news/second-data-release-of-the-next-generation-transit-survey.html. The processed lightcurves are available from the authors upon reasonable request. The Monte-Carlo models are available from the authors, upon reasonable request. The authors declare no competing interests. 


\begin{tabular}{|c|c|c|c|c|c|c|c|c|c|c|c|}
\hline Sp Type & NGTS ID & GAIA ID & TIC ID & RA & Dec & Mass $\left(M_{\odot}\right)$ & Radius $\left(R_{\odot}\right)$ & Luminosity $\left(L_{\odot}\right)$ & Distance $(\mathrm{pc})$ & Approximate Flare Rate per Hour & Magnitude \\
\hline M0V & NGTS J233315.1-385757 & 6538313140873424640 & 224245757 & 353.312913 & -38.965817 & 0.487596 & 0.489513 & 0.04772778 & 100.33 & 0.0123635 & 13.10 \\
\hline M0V & NGTS J045221.8-312424 & 4874911889552910000 & 1310695 & 73.090834 & -31.406834 & 0.597557 & 0.611732 & 0.07879962 & 137.421 & 0.0154544 & 13.26 \\
\hline M0V & NGTS J052346.3-361114 & 4822374303400198144 & 167745038 & 80.94287 & -36.187338 & 0.566358 & 0.574022 & 0.07396496 & 123.332 & 0.00618174 & 13.04 \\
\hline M0V & NGTS J061346.1-362248 & 2885025813007881728 & 267248553 & 93.442125 & -36.380098 & 0.585147 & 0.596407 & 0.07490093 & 137.363 & 0.0123635 & 13.24 \\
\hline M0V & NGTS J061054.6-370701 & 2884885281677800448 & 300200809 & 92.72739 & -37.116954 & 0.575812 & 0.585163 & 0.06398756 & 112.406 & 0.0401813 & 12.99 \\
\hline M1V & NGTS J233248.3-382456 & 6538532356004046592 & 224244565 & 353.201262 & -38.415564 & 0.542372 & 0.546805 & 0.05344092 & 90.7535 & 0.0123635 & 12.97 \\
\hline M1V & NGTS J051250.6-361938 & 4821058497219315328 & 14173066 & 78.210928 & -36.327354 & 0.596268 & 0.61012 & 0.06084004 & 201.14 & 0.0123635 & 14.16 \\
\hline M1V & NGTS J052652.1-373123 & 4821222942926810752 & 192785958 & 81.717213 & -37.523125 & 0.567364 & 0.575197 & 0.05580399 & & 0.00309087 & 13.57 \\
\hline M1V & NGTS J235034.7-373312 & 2310510165491596672 & \begin{tabular}{|l|l|}
183536494 \\
\end{tabular} & 357.644544 & -37.553375 & 0.506923 & 0.509045 & 0.04646323 & 83.9291 & 0.00927262 & 12.84 \\
\hline M1V & NGTS J111257.7-331216 & 5403344977522967424 & 23438898 & 168.240279 & -33.20455 & 0.460039 & 0.462724 & 0.03412572 & 73.1095 & 0.0185452 & 12.92 \\
\hline M2V & NGTS J045136.3-321720 & 4874656837214833664 & 1309522 & 72.901424 & -32.288803 & 0.56 & 0.806538 & 0.1124733 & 247.955 & 0.0278178 & 14.32 \\
\hline $\mathrm{M} 2 \mathrm{~V}$ & NGTS J050254.6-352000 & 4825066629419253632 & 1526841 & 75.72729 & -35.333409 & 0.469279 & 0.471581 & 0.03436859 & 69.7222 & 0.0185452 & 12.72 \\
\hline M2V & NGTS J051926.5-253444 & 2957763042671388416 & 30960826 & 79.860208 & -25.578811 & 0.460925 & 0.463568 & 0.03302738 & 90.6288 & 0.00927262 & 13.49 \\
\hline M2V & NGTS J053614.4-353309 & 4821870486556489216 & 24612475 & 84.059908 & -35.552443 & 0.501857 & 0.503859 & 0.03897486 & 90.6101 & 0.0154544 & 13.2 \\
\hline M2V & NGTS J062005.7-372555 & 5575203489668007936 & 393481864 & 95.023721 & -37.431899 & 0.400268 & 0.407808 & 0.02305492 & 87.7167 & 0 & 13.83 \\
\hline $\mathrm{M} 2.5 \mathrm{~V}$ & NGTS J045008.8-362401 & 4818804257863710336 & 77369893 & 72.536742 & -36.400372 & 0.545633 & 0.550421 & 0.05525498 & 118.868 & 0.0494539 & 13.07 \\
\hline $\mathrm{M} 2.5 \mathrm{~V}$ & NGTS J050359.5-305327 & 4876285488813663232 & 1439071 & 74.755373 & -30.999599 & 0.456069 & 0.458954 & 0.02748212 & 54.6863 & 0.00618174 & 12.8 \\
\hline $\mathrm{M} 2.5 \mathrm{~V}$ & NGTS J045901.2-305958 & 4875598534564509312 & 1535810 & 75.997888 & -30.890807 & 0.380031 & 0.389898 & 0.01965184 & 68.7496 & 0.0710901 & 13.65 \\
\hline $\mathrm{M} 2.5 \mathrm{~V}$ & NGTS J050810.8-371850 & 4823535318959536256 & 14084620 & 77.044884 & -37.313753 & 0.514811 & 0.517217 & 0.03755042 & 59.1582 & 0.0309087 & 12.29 \\
\hline $\mathrm{M} 2.5 \mathrm{~V}$ & NGTS J061516.4-360818 & 2885223381503521536 & 267327257 & 93.818249 & -36.138454 & 0.496041 & 0.497965 & 0.03437556 & 118.666 & 0.0278178 & 13.99 \\
\hline M3V & NGTS J035219.1-311459 & 4886786408973741568 & 166804322 & 58.079759 & -31.249846 & 0.544884 & 0.549588 & 0.03602405 & 123.566 & 0.0216361 & 13.63 \\
\hline M3V & NGTS J050230.0-355301 & 4824660428592359552 & 13982951 & 75.624229 & -35.883643 & 0.308218 & 0.327511 & 0.01185441 & 41.1162 & 0.00927262 & 13.1 \\
\hline M3V & NGTS J051925.5-235535 & 2958246827787260032 & 30961390 & 79.856241 & -23.926311 & 0.564934 & 0.572365 & 0.04099549 & 131.705 & 0.0865444 & 13.94 \\
\hline M3V & NGTS J052116.1-322429 & 4826608831916384640 & 78053729 & 80.317094 & -32.357958 & 0.401901 & 0.409266 & 0.01826787 & 39.3307 & 0.0494539 & 12.52 \\
\hline M3V & NGTS J000722.8-293528 & 2320750123439437184 & 12418184 & 1.845141 & -29.5912 & 0.22872 & 0.257223 & 0.007069197 & 40.1093 & 0.0216361 & 13.44 \\
\hline M4V & NGTS J035624.7-311140 & 4886831592030178944 & 166869904 & 59.102836 & -31.194413 & 0.402508 & 0.409808 & 0.01474621 & 37.7653 & 0.10509 & 12.7 \\
\hline M4V & NGTS J044312.0-322643 & 4874430475258301184 & 170882537 & 70.800028 & -32.445142 & 0.512283 & 0.514585 & 0.02822354 & & 0.0834535 & 12.6 \\
\hline M4V & NGTS J045519.0-321222 & 4873878176823736192 & 1357792 & 73.829291 & -32.206129 & 0.170431 & 0.201449 & 0.003005163 & 21.8796 & 0.0865444 & 13.72 \\
\hline M4V & NGTS J050423.8-373021 & 4823476460727785728 & 14001734 & 76.099199 & -37.505698 & 0.434448 & 0.438763 & 0.02066878 & & 0.148362 & 12.42 \\
\hline M4V & NGTS J2341092-363819 & 2311548448064869120 & 224276435 & 355.288136 & -36.638609 & 0.253768 & 0.279818 & 0.005977345 & 38.7917 & 0.0649083 & 13.79 \\
\hline
\end{tabular}

Table 5. The Spectral type, NGTS identifier, Gaia source ID, Tess Input Catalog (TIC) ID, RA, Dec, Stellar Mass (in Solar mass units), Stellar Radius (in Solar radi units), Distance (in parsecs), Stellar Luminosity (in Solar luminosity units), and the Macroscopic Flare Rate (per hour) for the stars used in the analysis. The Stellar masses, radi. and luminosity data is from the Tess Input Catalog release V8. ${ }^{42}$ 